\title{
Management of Enteroatmospheric Fistulae with Grade 4 Open Abdomen In Addition Short Bowel Syndrome, In Frozen Abdomen Also Due To These, the Secondary Problems. A New Surgical Technique for Closure of Entreroatmosoheric Fistulae in Frozen Abdomen: Anastomosis by Benefiting the Mucosal Hypertrophy
}

\author{
Mustafa Yavuz \\ Department of General Surgery, State Hospital, Nevsehir (Capadoccia), Turkey \\ E-mail: mustipyavuz@hotmail.com
}

\begin{abstract}
Grade 4 open abdomen is usually result of abdominal infection, sepsis usually because of (EAF)enteroatmospheric fistula. EAF and small bowel with open abdomen are a rare status and one of the biggest problem of surgeon, especially in frozen abdomen. The patients, have all these problems, rarely continue to their life. In a short time they usually die. It is very difficult, these patients to go on their lifes. Many surgeons always try to develop new techniques, approaches and at the past many of them tried but there are no fixed algorithmys, treatment options are determined. For all these problems, especially in frozen abdomen we developed a new technique by benefitting the past experiments of the surgeons and approaches also knowledges about these issues. Negative pressure wound therapy (NPWT) has been used affectively and every kind of literature knowledges about intestinal fistula, short bowel, nutrition. A patient had undergone multiple operations at the different times and because of these had EAF were treated with this technique. We managed the closure of fistula but we could not preserve her from the secondary problems more than 1,5 years during process. According to me, this case, this experiment must be learned by all associations of surgery.
\end{abstract}

Key words: EAF; Enteroatmospheric fistula; Negative pressure wound therapy; NPWT; Open abdomen; Nutrition; Mucosal flap; Mucosal hypertrophy

DOI: $10.7176 / \mathrm{JHMN} / 83-03$

\section{Introduction}

An enteroatmospheric fistula (EAF), defined as a connection between the atmosphere and an intestinal lumen, is a major complication in patients who are treated with an open abdomen (OA), and also itself is a reason of an open abdomen. EAF is caused by some factors, including trauma, iatrogenic effects (surgical or percutaneous drainage), infection, radiation, inflammatory bowel diseases and malignant diseases (1). We usually face this problem after the major surgicals and recurrent surgicals.

However, open abdomen (OA), a closure problem of peritoneal cavity and as a term, first used by Ogilvie in 1940, is currently applied usually management of three clinical conditions by surgeons: (a) damage control surgery for several abdominal trauma, (b) damage control surgery for severe abdominal sepsis (source control-temporary drainage), also (c) management of abdominal compartment syndrome. Surgeons face various problems in the management of the open peritoneal cavity, such as upper examples. Open abdomen (OA) is suitable for providing damage control thus the abdomen can be re-evaluated, making re-intervention, and it leads, intervention is possible, if necessary. In addition, early closure of the OA is not always an easy task for surgeons, and usually it can cause morbidity, including intestinal fistulisations and giant hernia $(2,3,4,5)$.

Short bowel syndrome is a serious and complex disease also condition that impairs quality of life. It might lead life-threatening complications. The main problems are always related with fluid, electrolyte and nutrient balances. Short bowel syndrome, defined insufficient absorption of fluid, electrolytes and nutrients, usually are caused by having abdomen surgeries(mesenteric embolism, traumas, inflammatory bowel diseases, intestinal malignant diseases) as EAF, OA. Also without having any surgery, due to some bowel absorption diseases, we can see this syndrome in several cases. Only short 
bowel is defined as that; having less 40 or $60 \mathrm{~cm}$ bowels or dependence to total parenteral nutrition for 42 days and more $(3,4,5)$.

Frozen abdomen is an other condition that we usually see after severe abdominal surgeries and recurrent surgeries also radiotheraphies(for abdominal malignants). It is defined by dense brids and also it is a serious problem for surgeons, having a frozen abdomen, if they have to make any abdominal operation. To make any abdominal operation usually leads to OA or EAF.

Beyond upper subjects, a very challenging task for the surgeon dealing with a case having all these upper problems. It must be known in detail the available and effective techniques for temporary abdominal closure in order to manage the OA with other problems during the first weeks. Furthermore, the patients have to be treated in a surgical intensive care unit (SICU) with relative experience in managing specific problems and complications related to patients with all these problems, namely, severe dehydration due to excessive fluid losses from the exposed surface of the OA and EAF, hypercatabolism associated with the underlying disease, electrolyte and acid-base disturbances, septic complications, and the most dreaded complication, the development of an other enteroatmospheric fistula (EAF)as well as $(5,6)$.

\section{Patient}

A 60 years old female patient had undergone multiple operations due to colon cancer. She had grade 4 $\mathrm{OA}$, high level EAF and due to these liquid-electrolyte imbalance also skin burns, caused from the bile secrection furthermore frozen abdomen and partly septic condition. She had end colostomy made from transvers colon due to rectovaginal fistulae caused by radiotherapy. She was suffering from all these. First we took her to intensive care unit and started to correct the liquid-electrolyte imbalance at the same time the septic condition. We gave total parenteral nutirition(TPN) with omega 3, alanil-glutamin including solutions and trace elements from the central venosus catheter (6-7). According to the blood culture received that before was given antbiotherapy. Reoperation was impossible because of frozen abdomen and heavy adhesions. According to radiologic measurements, at the distance nearly $60 \mathrm{~cm}$ from the treitz ligament, there was whole seperated small bowel from its distal one. The distance between seperated small bowels were nearly $10 \mathrm{~cm}$ and these ones were adhesive to around tissues.

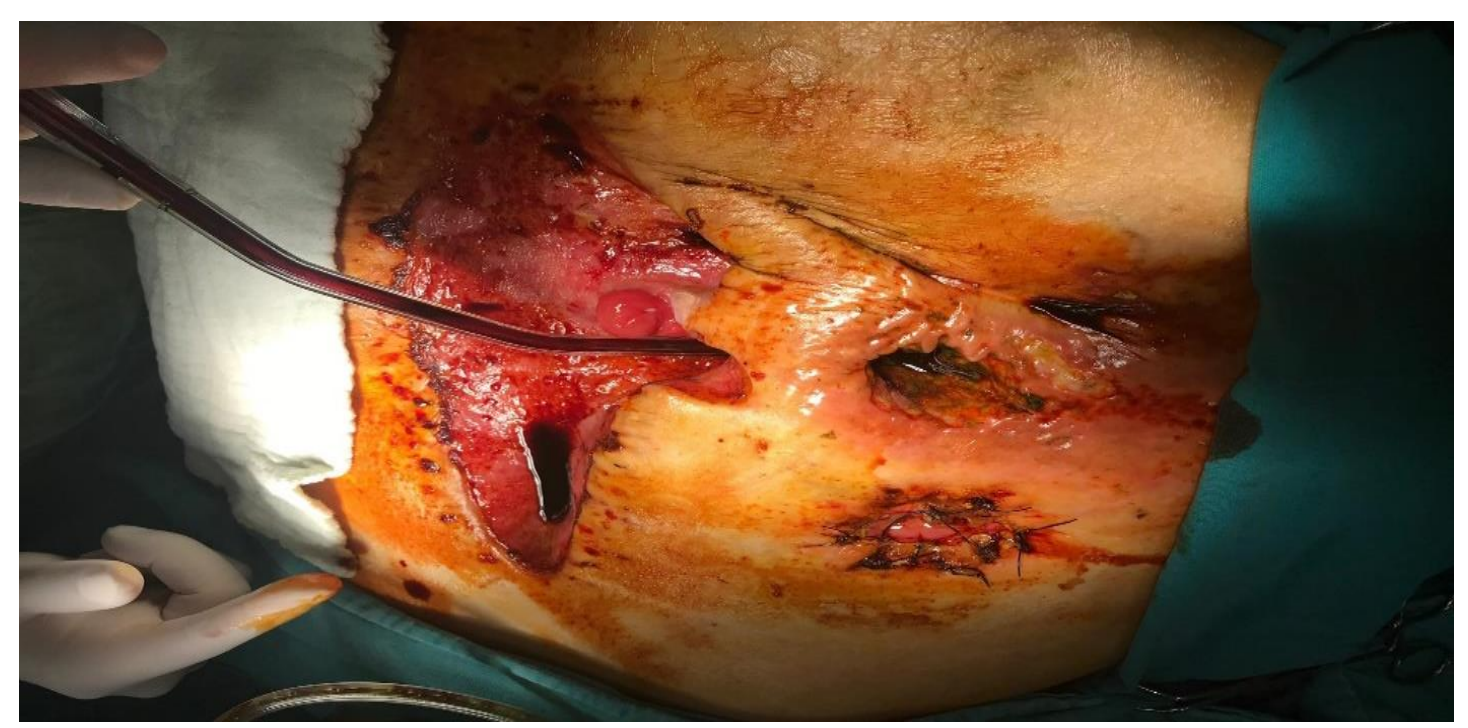

Figure 1 The first status of the EAF and grade 4 open abdomen

Treatment

We had to protect the skin and heal the sepsis thereby do better the delirium cause of these. The patient had to be rehabilitated, mobilized and continued his daily life, at least partially, we began to oral intake to provide gastrointestinal physiology, but due to insufficient absorption due to the short bowel, we continued antibiotherapy and nutritional support, trace element supplementation. To reduce the wound, protect the skin and control intestinal secretion, we created an artificial stoma with negative pressure application, and we repeated this application for about 4-5 days at varying intervals as done in the literatüre. It did not rescue the patient from total parenteral nutrition (tpn) but controlled sepsis, provided comfort and rehabilitation $(6,7,8)$. figure 2,3 


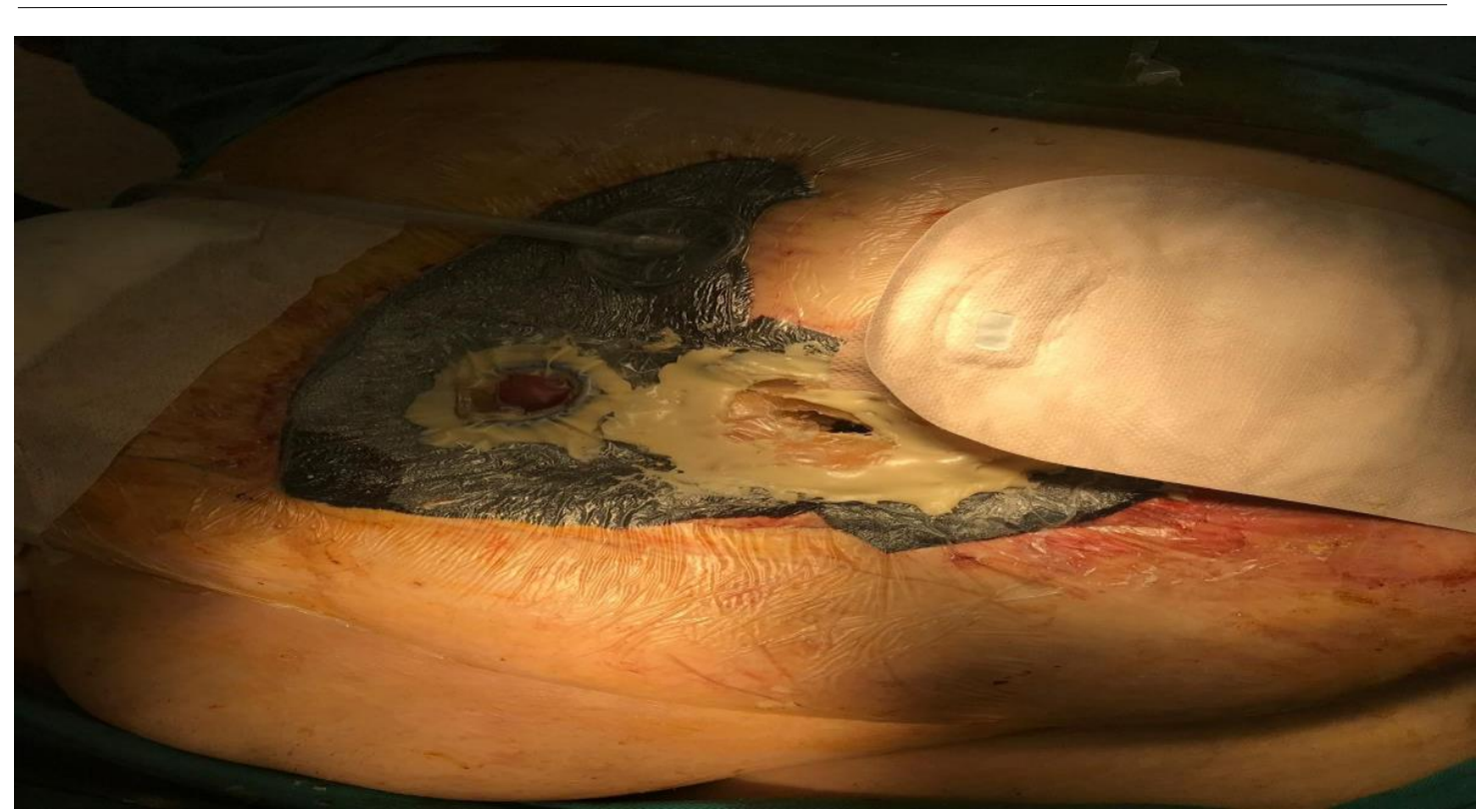

Figure 2 NPWT approaching with end colostomy, distal and short proximal small bowels by making artificial ileostomy

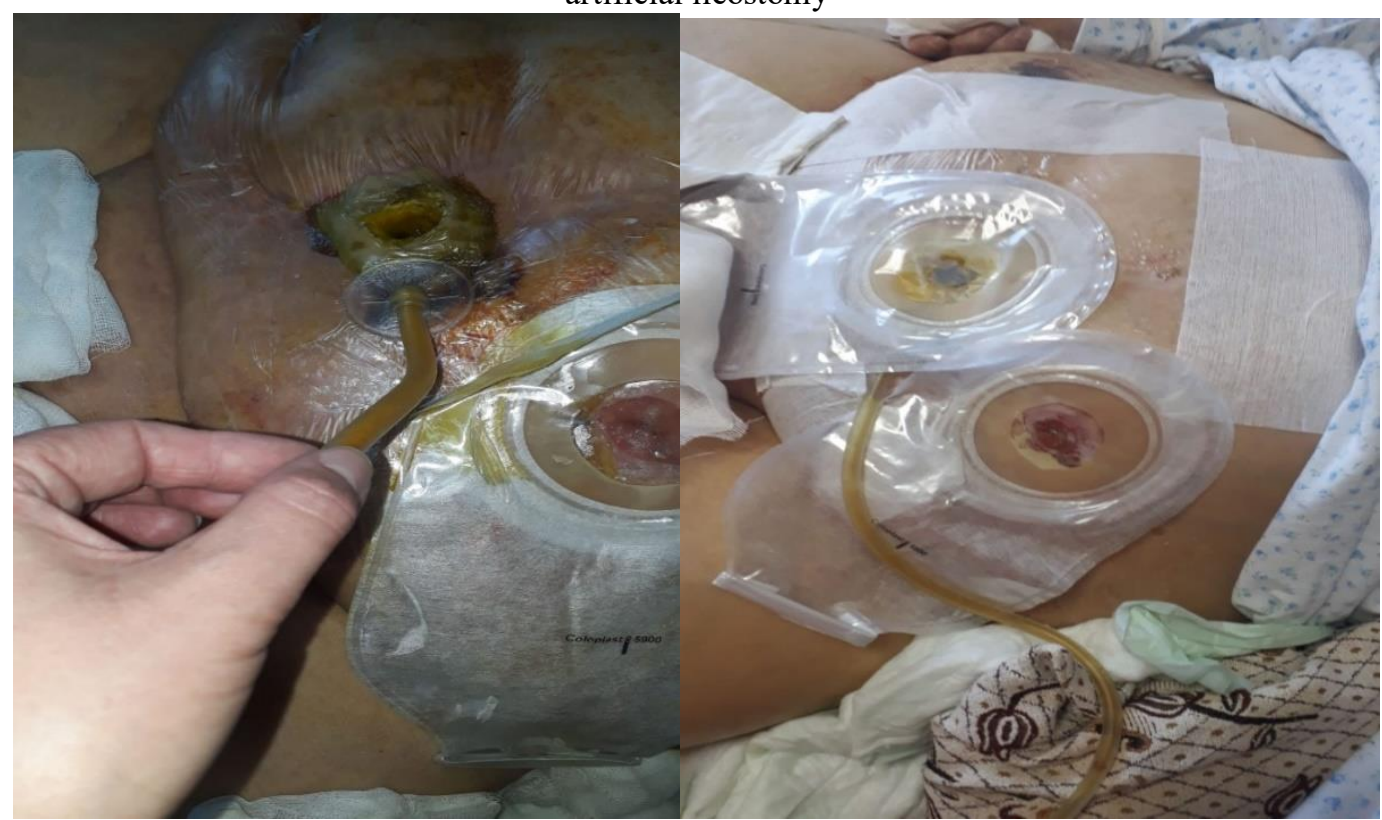

Figure 3 Step by step closed open abdomen, end colostomy and one ileostomy by taking advantage of mucosal hypertrophy adducted proximal- distal bowels

We continued the same treatment for about a year, the wound got smaller but the brides did not soften, and we had to continue with TPN and trace element therapy due to the ongoing short bowel.This caused cholelithiasis sometimes cholangitis, one of the problems related to the TPN, and we did ercp for the occasional cholangitis attacks. However, because of the adhesion, cholecitectomy was impossible as it would create new injuries. Reoperation was impossible to close the fistula, the loops were far apart. A new formula was needed, all similar cases in the literature were screened, there was no same situation or patients had been lost in a short period of time within 6 months. Mucosal hypertrophy in the proximal bowel due to chronic fistula gave an idea. Mucosal hypertrophy almost closed the $10 \mathrm{~cm}$ dissociated distance If we could combine it, we could use this hypertrophic mucosa as a graft. If the fistula was cut, the skin that was almost closed with the vac would cover the graft and both the fistula and the skin would be closed. In the literature, mucosal hypertrophy in fistula intestines is a known condition, but it has never been used for fistulas, cyanoacrylate, the silicone fistula plug (1) method has been used, but where they are used, there are no fully dissociated bowels or without serosa 
intestines. We anastomosed the circular hypertrophic proximal mucosa to the distal ans with a 3-0 double needle vicrile, applied cyanoacrylate on it and closed with a circular drape. For 3 days no leak occurred. We started oral intake at the 4th day and there was no problem. 5th day we increased oral intake and 6th day we were sure about leak. Unfortunately cholangiocepsis did not improve and the patient's condition worsened in the following days, but the fistula was closed and the skin was about to close. figure 4

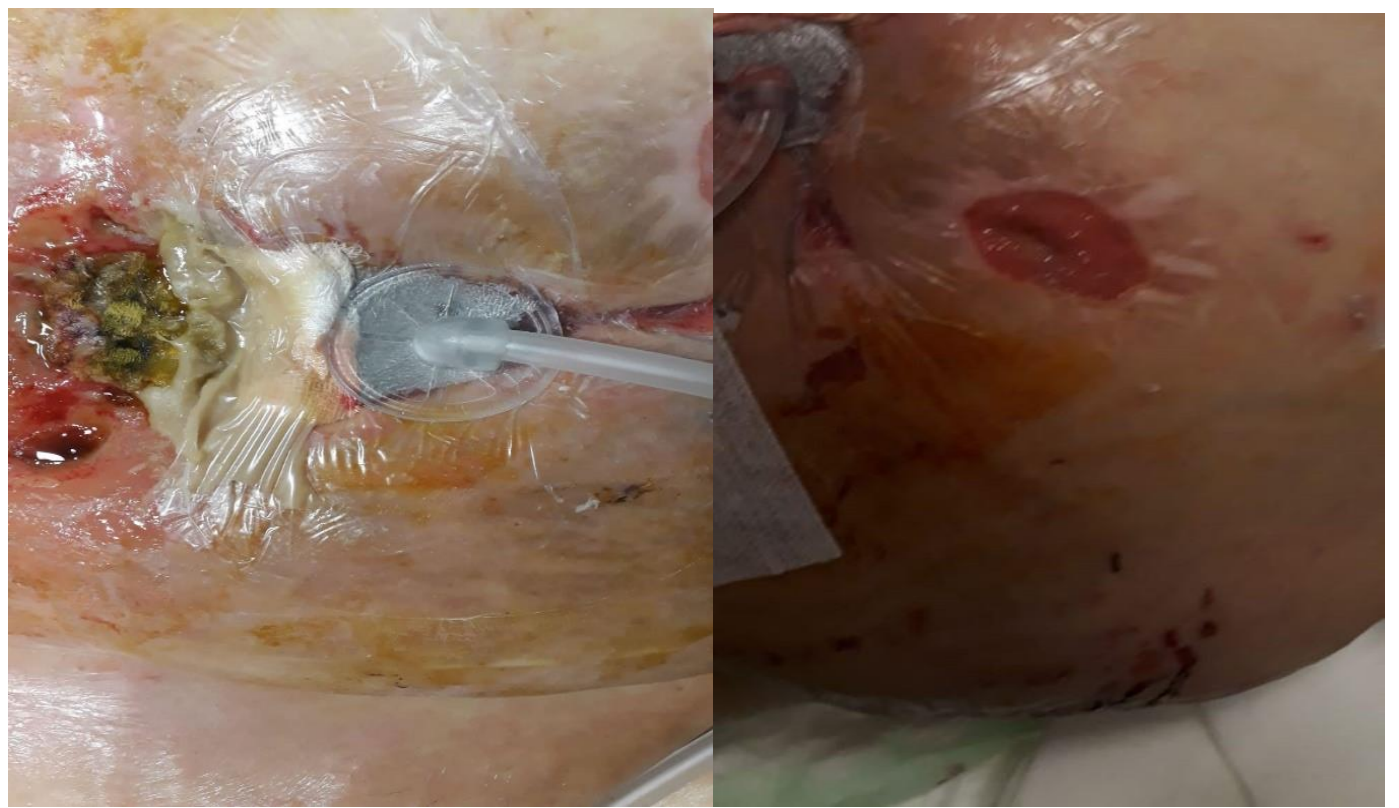

Figure 4 Closed EAF, end colostomy and about to close stomach wound(OA)

Conclusions

Consensus working definition for EAF in OA with short bowel syndrome seen rarely have been proposed in this review to help the surgeons manage patients with these conditions together or one of these, at the same time, to help evalution of novel techniques for these conditions. Fistula prevention is essential in the management of OA. If an EAFdevelops, it should be recognised as early as possible. Although EAFs in OA with short bowel syndrome seen rarely will continue to be a challenge for surgeons. Applying a negative pressure is a safe and reliable way to manage EAF in OA with short bowel. Multidisciplinary approach and acknowledge are very important to manage these patients. So we share our experience as a novel technic by combination of literature in treating EAF in OA with short bowel syndrome presents gracious results if you can make it earlier.

\section{References}

1. M Tahir Ozer, Hüseyin Sinan, Nazif Zeybek \& Yusuf Peker, A simple novel technique for enteroatmospheric fistulae: silicone fistulae plug, International Wound Journal ISSN 1742-4801, 2014

2. Cem Terzi, Tufan Egeli, Aras E Canda \& Naciye C Arslan, Management of enteroatmospheric fistulae, International Wound Journal ISSN 1742-4801, 2014

3. Çelik A., Bağırsak yetmezliği/kısa bağırsak sendromunda transplant dışı cerrahi yaklaşımlar, Çocuk Cerrahisi Dergisi 31(Ek say1):56-76, 2017 doi:10.5222/JTAPS.2017.1012

4. Bjarnason T, Montgomery A, Hlebowicz $\mathrm{J}$ et al: Pressure at the bowel surface during topical negative pressure therapy of the open abdomen: An experimental study in a porcine model. World J Surg 2011;35:917-923. 
5. A. Marinis, G. Gkiokas, E. Argyra, G. Fragulidis, G. Polymeneas, D. Voros, "Enteroatmospheric Fistulae"-Gastrointestinal Openings In The Open Abdomen: A Review And Recent Proposal Of A Surgical Technique, Scandinavian Journal of Surgery 102: 61 -68, 2013

6. Zhou X, Li YX, Li N, Li JS. Glutamine enhances the gut-trophic effect of growth hormone in rat after massive small bowel resection. J Surg Res 2001;99:47-52. https://doi.org /10.1006/jsre.2001.6108

7. Gu Y, Wu ZH, Xie JX, et al. Effects of growth hormone (rhGH) and glutamine supplemented parenteral nutrition on intestinal adaptation in short bowel rats. Clin Nutr 2001;20:159-166.

8. D’Hondt M, Devriendt D, Van Rooy F, Vansteenkiste F, D’Hoore A, Penninckx F, Miserez M. Treatment of small-bowel fistulae in the open abdomen with topical negative-pressure therapy. Am J Surg 2011;202:e20-4. 